\title{
EDITORIAL
}

Anton Nijholt · Toyoaki Nishida

\section{Social intelligence design for mediated communication}

Published online: 19 August 2005

(C) Springer-Verlag London Limited 2005

This Special Issue of AI and Society contains a selection of papers presented at the 3rd Social Intelligence Design Workshop held at the University of Twente, Enschede, the Netherlands, in July 2004.

Social Intelligence Design as a research field attempts to integrate understanding and designing social intelligence, as identified in the first workshop held in Matsue, Shimane, Japan, in 2001 (Nishida 2001). On the one hand, it involves engineering approaches concerning design and implementation of systems and environments, ranging from group/team oriented collaboration support systems that facilitate common ground building, goal-oriented interaction among participants to community support systems that support large-scale online discussion. On the other hand, it involves scientific approaches addressing cognitive and social psychological understanding of social intelligence, and provides a means for predicting and evaluating the effect of a given communication medium on the nature of discussions, interaction dynamics, and conclusions. In addition, it encompasses pragmatic considerations from economy, sociology, ethics and many other disciplines, for social intelligence design has a direct relation with the society. The engineering and analytical approaches are complementary to each other and should be integrated intimately, for good systems cannot be built without good understanding and vice versa. ${ }^{1}$

Clearly, as mentioned in Fruchter et al. (2005), "The central idea here is social intelligence. Conventionally, social intelligence has been discussed as an ability of an actor/agent to relate to other actors/agents in a society, understand them,

\footnotetext{
A. Nijholt $(\square)$

Centre of Telematics and Information Technology (CTIT), University of Twente, PO Box 217, 7500 AE,

Enschede, The Netherlands

E-mail: anijholt@cs.utwente.nl

T. Nishida

Graduate School of Informatics, Kyoto University, Yoshida-Honmachi, Sakyo-ku, Kyoto 606-8501, Japan

E-mail: nishida@i.kyoto-u.ac.jp
}

\footnotetext{
${ }^{1}$ See http://www.ii.ist.i.kyoto-u.ac.jp/sid/ for the series of the Social Intelligence Design workshops
} 
and interact effectively with them. It is contrasted with other kinds of intelligence such as problem solving intelligence (an ability to solve logically complex problems) or emotional intelligence (an ability to monitor one's own and others ${ }^{6}$ emotions and to use the information to guide one's thinking and actions). Alternatively, social intelligence might be attributed to a collection of actors/ agents and defined as an ability to manage complexity and learn from experiences as a function of the design of social structure. This view emphasizes the role of social conventions that constrain the way individual agents interact with each other. A desirable social culture will afford the members of the community to learn from each other".

The second workshop was centred on developing theories to enhance the understanding and conceptualization of human cognition and interpersonal interaction to underpin the practice of Social Intelligence Design in order to explain how social intelligence is created and maintained through personal/ human contact. We discussed how a contact could be facilitated by the design of mediated settings and the impact of the social intelligence perspective on the design of user interfaces. We argued for a change in the paradigm for interface design towards the view of the user interface as a channel for communication between remote participants.For the 2004 workshop we distinguished the following four themes:

- Natural Interactions - covering theory, modelling and analytical frameworks that have been developed with Social Intelligence Design in mind, including situated computation, embodied conversational agents, sociable artefacts, socially intelligent robots.

- Communities - covering community media, communication patterns in online communities, knowledge creating, network and anonymous communities.

- Collaboration Technologies and tools - covering innovations to support interactions within communities, covering a range from knowledge sharing systems, multi-agent systems and interactive systems.

- Application Domains - including design, workspaces, education, e-commerce, entertainment, digital democracy, digital cities, policy and business.

From these four areas we also derived the three categories of papers in this Special Issue (see below). As has been done in the previous Special Issue of this journal (AI and Society 2005, Issue 19, Editorial) devoted to the Social Intelligence Design workshop of 2003, we will shortly introduce the areas and the selected papers in these areas in order to explain their contribution from the point of view of the development of Social Intelligence Design we sketched in this editorial.

\section{Natural interaction: virtual humans and robots as conversational agents}

We start this special issue with two papers that explicitly address social intelligence issues in face-to-face communication. The communication that is considered is that between human and virtual embodied agent(s) and between human and robot. In other papers in this issue, especially those dealing with collaboration technologies for distributed workspaces, it is discussed how tools 
and environments can be designed in such a way that despite the lack of natural face-to-face interaction social interaction can be supported by tools and environments.

One obvious way to embed social intelligence into a human-computer interface is the use of embodied conversational agents. Yamashita et al. emphasize the role agents can play, in addition to the embodiment, to more effectively communicate that have been made available by members in a web-based community. Each opinion is available as text, but rather than have this presented as text or read aloud by text-to-speech synthesis, it can be transformed into a conversation between two animated agents shown on the screen. In this paper two experiments are discussed. In these experiments the effect of transforming text into conversational form on comprehension is studied. The effect was studied both for short sentences and for long sentences. Moreover, the knowledge level of the participants in the experiments was taken into account. As shown in the paper, the basic hypothesis that this transformation to a conversational form promotes comprehension (especially for long sentences and persons lacking relevant knowledge) was supported. Although it is not addressed explicitly in this paper, one may also expect that users will find the system more enjoyable than in the non-conversational case.

Users can communicate with virtual, embodied agents that inhabit a virtual 2 or 3D world, as in the previous paper. There are of course also situations where humans are expected to communicate with physical agents, robots for example. Do people communicate as easily to these agents as, in general, to their fellow humans? It is well known that humans attribute human characteristics to systems they can interact with. Clifford Nass and others have made this clear in many experiments, introducing the Computers Are Social Actors (CASA) paradigm. And, as we can have positive and negative attitudes towards certain people or certain kinds of people, we can as well expect that we will have different attitudes towards different types of agents (virtual or physical), depending on their appearance and their (expected) behaviour. Nomura et al. investigate factors that prevent social interaction with robots. An important tool in their research is the so-called Negative Attitude towards Robots Scale. Using this scale, experiments have been performed where "scaled" subjects were asked to approach a robot, talk to the robot, answer questions posed by the robot and touch the robot. Depending on their measured attitudes towards robots different behaviours could be distinguished. In addition it was studied whether taking into account gender and previous experiences with robots had influence on the results. Results of this type of research may help to make decisions in robot design that anticipate possibly negative attitudes of their human conversational partner.

\section{Communities, collaboration and social interaction}

Although the terminology may differ, the following three papers are about the design of distributed workspaces and all the three tackle the problem of how to allow and support, despite being distributed in space - and sometimes also in time - social interaction that we normally see and find useful in a joint physical workspace (office, class room, meeting room, workshop, et cetera). 
Advanced information and communication technology allows workers to cooperate in distributed workspaces using audio and video channels and shared electronic text and drawings. Haptics and virtual reality environments can make these workspaces and cooperation within them even more realistic. Whether advanced or simple, traditional or non-traditional, when looking at social intelligence design similar issues need to be addressed. In the papers mentioned below we see efforts to manage and improve "presence" in distributed workspaces, efforts to understand and support discourse behaviour in distributed collaborative settings, and a study on the effects of using virtual workspaces on the social functioning of individuals, groups and organizations.

Presence, from the point of view of awareness of others sharing the workspace or the environment, is one of these issues. Hofte et al. paid attention to what they call presence technology. This community technology makes it possible to determine who is available for communication, with whom can we exchange information, and which co-workers are available in the joint workspace. Negotiating conversational availability is one of the aspects addressed in this research. Trust and privacy are among the other issues that are investigated in this research. A trust model is introduced and the notion of presence is related to location and virtual distance. Prototypes and experiments with users in virtual workspaces are presented.

Fruchter and Cavallin discuss the communication process of the building design process when mediated by computers. They investigate data obtained from direct observation, video recording, and screen capturing (from PCs and notebooks used by team members collaborating in the design process). In addition to traditional discourse research, here both the physical setting of the interactions, the workspace as well as the psychosocial aspects of the interactions are taken into account. Interesting findings such as faked pointing (a communication failure situation caused by wrong assumptions about the contents that are transmitted with the interface which may lead to misunderstanding and delays in the communication process) are identified and analysed in terms of visibility and awareness.

Pumareja and Sikkel discussed the effect of long-term use of distributed workspaces on the social functioning of individuals, groups, and organizations. Social interaction in an organization is discussed in the context of long-term use of groupware systems. The framework that is used to investigate this topic is that of social constructivism. Apart from theoretical observations the authors introduce a case study where a distributed group of educators provide training and consultation in the domain of special education and orthopedagogy. One of the conclusions is that a collaborative system can become structurally assimilated into the collaboration and collaborative processes of the users.

\section{Applications}

Below we discuss three more papers in this special issue. In most of the previous papers the research had already been presented in particular application domains. For example, the building design context where teams composed of architecture, engineering, and construction management students are engaged in distributed collaboration in the paper by Fruchter and Cavallin, or the educa- 
tional context, where geographically dispersed educators need to collaborate in an institute of higher education in the paper by Pumareja and Sikkel. In the following three papers we see some more areas and domains where attempts and suggestions are presented to incorporate social intelligence, both from the individual and from the community point of view, into systems that support human activities.

One activity all of us are familiar with is attending meetings. Rather than looking at communication problems that arise when participants present in different locations meet and collaborate, the paper by Nijholt et al. ("Meetings and Meeting Modelling in Smart Environments") is about modelling the meeting process and meeting behaviour during traditional face-to-face meetings where people sit around a meeting table and discuss agenda matters. The paper describes research that is performed in the context of two large European projects on capturing, modelling, and understanding of activities in a smart meeting room. In the first of these projects [Multi-Modal Meeting Manager (M4)], meeting acts and sequences of meeting acts are defined and machinelearning methods are used to have the environment learn to recognize them. This research aims at building a system that allows off-line retrieval and browsing of meeting information. A more recently started successor project [Augmented Multi-party Interaction (AMI)] that is discussed much more takes into account more individual meeting behaviour, including emotions and social intelligence aspects of interactions, and has also a modest aim at real-time support of meeting participants (including possibly remote meeting participants) during a meeting. An interesting test for a system of being able to understand what is going on in a meeting room is the regeneration of parts of a meeting from the captured and (ultimately automatically) annotated multimodal information in different ways. For example, as an answer to an information query or as a request for a summarization.

Miura et al. discuss the role of pre-existing domain knowledge of the searcher in information retrieval. In the paper retrieval actions, e.g. selection of keywords or operations on web pages, are categorized and used to study and compare information retrieval behaviour between those who have satisfactory task-related knowledge and those who have not. Experiments using think-aloud sessions were performed and their results are discussed. This leads to useful observations on the relationship between retrieval behaviour and preexisting knowledge, as it can be available in a community of users. Some strategies making use of existing community knowledge and future systems for information retrieval are suggested, but for the moment are left for future research.

Finally, Blake and Tucker are concerned with the question about how social intelligence and interaction techniques can compensate for poor communicating across the "Digital Divide", i.e. the gap between those who have unlimited access to the information society and those who have poor and limited access to this society because of personal handicaps, poor information structure, cultural bias, and poor facilities. How can computer-based artefacts act as communication bridges? And, more importantly, how should we design our IT applications, our software systems and our interfaces in such a way that they can deal with this gap? The authors discuss field trials that are underway in remote rural regions in South Africa in Tele-Health projects. It is concluded that especially in 
a situation with poor facilities and infrastructure several aspects of social intelligence influence users and system use and therefore need to be dealt with in order to allow successful use of computer-based artefact in these situations.

\section{References}

Blake E, Tucker W User interfaces for communication bridges across the digital divide (in this volume)

Fruchter R, Nishida T, Rosenberg D (2005) Understanding mediated communication: the social intelligence design (SID) approach. AI Soc 19:1-7

Fruchter R, Cavallin H Developing methods to understand discourse and workspace in distributed computer mediated interaction (in this volume)

Hofte G, Mulder I, Verwijs C Close Encounters of the virtual kind: a study on place-based presence (in this volume)

Miura A, Fujihara N, Yamashita K Retrieving information on the World Wide Web: Effects of domain specific knowledge (in this volume)

Nijholt A, Heylen R, Op den Akker D Meetings and meeting modelling in smart environments (in this volume)

Nishida T (2001) Social intelligence design - an overview. In: Terano T, Nishida T, Namatame A, Ohsawa Y, Tsumoto S, Washio T (eds) Exploring new frontiers on artificial intelligence - selected papers from the first international workshops of Japanese society of artificial intelligence, December 2001. Lecture notes on artificial intelligence LNAI2253. Springer, Berlin Heidelberg New York

Nomura T, Kanda T, Suzuki T. Experimental investigation into influence of negative attitudes toward robots on human-robot interaction (in this volume)

Pumareja D, Sikkel K. Getting used with groupware - a first class experience (in this volume)Yamashita K, Kubota H, Nishida T. Designing conversational agents: effect of conversational form on our comprehension (in this volume) 